\title{
Composição, diversidade e distribuição geográfica de plantas vasculares de uma Floresta Ombrófila Densa Atlântica do Sudeste do Brasil
}

\author{
Renato Augusto Ferreira de Lima ${ }^{1,5}$, Vinícius Castro de Souza ${ }^{2}$, \\ Vinícius Antonio de Oliveira Dittrich ${ }^{3}$ \& Alexandre Salino ${ }^{4}$ \\ ${ }^{1}$ Programa de Pós-graduação em Ecologia, Instituto de Biociências - IB, Universidade de São Paulo - USP, \\ Rua do Matão, 321, CEP 05508-090, São Paulo, SP, Brasil, http://www.ib.usp.br \\ ${ }^{2}$ Departamento de Ciências Biológicas, Escola Superior de Agricultura 'Luiz de Queiroz' - ESALQ, \\ Universidade de São Paulo - USP, Av. Pádua Dias, 11, CP 9, CEP 13418-900, Piracicaba, SP, Brasil, \\ http://www.esalq.usp.br \\ ${ }^{3}$ Departamento de Botânica, Instituto de Ciências Biológicas, \\ Universidade Federal de Juiz de Fora - UFJF, Rua José Lourenço Kelmer, s/n, CEP 36036-900, \\ Juiz de Fora, MG, Brasil, http://www.ufjf.br/herbario \\ ${ }^{4}$ Departamento de Botânica, Instituto de Ciências Biológicas, Universidade Federal de Minas Gerais - UFMG, \\ Av. Antônio Carlos, 6627, CP 486, CEP 31270-901, Belo Horizonte, MG, Brasil, \\ http://www.icb.ufmg.br/icb/ \\ ${ }^{5}$ Autor para correspondência: Renato Augusto Ferreira de Lima, e-mail: raflima@usp.br
}

LIMA, R.A.F., SOUZA, V.C., DITTRICH, V. A. O. \& SALINO, A. Composition, diversity and geographical distribution of vascular plants of an Atlantic Rain Forest, Southeastern Brazil. Biota Neotrop. 12(1): http:// www.biotaneotropica.org.br/v12n1/en/abstract?inventory+bn01612012012

\begin{abstract}
In the Montane and Submontane Rain Forest of the Carlos Botelho State Park - PECB (ca. 37,000 ha) the composition, richness and geographical distribution of native, vascular forest species was evaluated. The analysis of 1143 species of 140 families supported the pattern found for other forests of Eastern Brazil, showing high species richness of Myrtaceae (85 species), Orchidaceae (81), Fabaceae (57), Asteraceae, Melastomataceae (54), Lauraceae (53), Rubiaceae (51), Bromeliaceae (43), Piperaceae (30) and Solanaceae (25), besides ferns (123). The most species-rich genera were Eugenia (34), Ocotea (26), Leandra, Myrcia, Vriesea (18), Piper, Solanum (16), Miconia (14), Mollinedia (13), and Peperomia (12). The richness and composition varied greatly among life forms, as well as the number of families represented in each one of them (only Rubiaceae had species in all life forms, except parasites). Trees had the largest contribution of total richness (39.1\%), a value that represented more than $20 \%$ of the species listed for the whole Atlantic Forest of Southeastern Brazil. Trees were followed by epiphytes $(22.4 \%)$, herbs $(18.4 \%)$, shrubs $(10.1 \%)$, lianas $(9.1 \%)$, and parasites $(0.9 \%)$. The overall richness and composition of life forms was quite close to other neotropical forests (e.g. high contribution of ferns among epiphytes), although some life forms remain undersampled in the PECB (mainly herbs, lianas and epiphytes). The occurrence of species endemic to the Atlantic Forest was pronounced (65\%), with a predominance of species restricted to the Southern Atlantic Forest (43\%). Pantropical species were rare (2\%), being more common among ferns. Myrtaceae and Melastomataceae were the families with greater number and proportion of endemic species. Keywords: Atlantic Forest, Carlos Botelho State Park, distribution patterns, life forms, species composition.
\end{abstract}

LIMA, R.A.F., SOUZA, V.C., DITTRICH, V. A. O. \& SALINO, A. Composição, diversidade e distribuição geográfica de plantas vasculares de uma Floresta Ombrófila Densa Atlântica do Sudeste do Brasil. Biota Neotrop. 12(1): http://www.biotaneotropica.org.br/v12n1/pt/abstract?inventory+bn01612012012

Resumo: Na floresta Ombrófila Densa do Parque Estadual de Carlos Botelho - PECB (ca. 37.000 ha) foi avaliada a composição, riqueza e distribuição geográfica das espécies vasculares nativas. A análise de 1143 espécies pertencentes a 140 famílias (sensu APG III) confirmaram o padrão encontrado para outras florestas do Leste do Brasil, com altas riquezas de Myrtaceae (85 espécies), Orchidaceae (81), Fabaceae (57), Asteraceae, Melastomataceae (54), Lauraceae (53), Rubiaceae (51), Bromeliaceae (43), Piperaceae (30) e Solanaceae (25), além de pteridófitas (123). Os gêneros mais ricos do Parque foram: Eugenia (34), Ocotea (26), Leandra, Myrcia, Vriesea (18), Piper (16), Solanum (15), Miconia (14), Mollinedia (13) e Peperomia (12). Houve grande variação na riqueza e composição de espécies entre hábitos, bem como no número de famílias representadas nos hábitos considerados (apenas Rubiaceae teve espécies em todos os hábitos, exceto parasitas). As árvores obtiveram a maior contribuição na riqueza total $(39,1 \%)$, valor que representou mais de $20 \%$ das espécies de árvores citadas para toda a Floresta Atlântica do Sudeste do Brasil. Em seguida, foram os epífitos (22,4\%), ervas (18,4\%), arbustos $(10,1 \%)$, lianas $(9,1 \%)$ e parasitas $(0,9 \%)$. De maneira geral, a riqueza e a composição por hábitos foram bastante similares ao encontrado nas demais florestas neotropicais (e.g. alta contribuição de pteridófitas ente os epífitos), apesar de alguns dos hábitos permanecerem subamostrados no PECB (principalmente ervas, lianas e epífitos). A ocorrência de espécies endêmicas da Floresta Atlântica foi elevada (65\%), com predominância de espécies de ocorrência no Sul da Floresta Atlântica (43\%). Espécies pantropicais foram raras (2\%), sendo mais comuns entre as pteridófitas e entre as herbáceas. Myrtaceae e Melastomataceae foram as famílias com maior número e proporção de espécies endêmicas.

Palavras-chave: Floresta Atlântica, Parque Estadual Carlos Botelho, padrões de distribuição, hábitos, florística. 


\section{Introdução}

A exuberância das florestas tropicais e subtropicais impressiona pesquisadores de todo o mundo há décadas. A diversidade de espécies, de formas de vida e de interações biológicas é tão grande que por vezes pequenas áreas entre os trópicos possuem diversidade comparável a vastas áreas temperadas. Áreas florestais no Equador e da Malásia, por exemplo, contém mais de 1.100 espécies lenhosas em poucas dezenas de hectares (Center for Tropical Forest Science 2008). Dentre as diferentes zonas biogeográficas do mundo, a flora Neotropical é uma das mais ricas, com estimativas em torno de 90.000 espécies (Gentry 1982). No Brasil, estimativas giram entre 45.000 e 60.000 espécies vasculares, ou seja, entre 15 e $20 \%$ de toda a flora mundial (Shepherd 2000). Mais de $97 \%$ destas se refere às angiospermas, dentre as quais as principais famílias são: Fabaceae sensu lato, Orchidaceae, Asteraceae, Poaceae, Euphorbiaceae, Myrtaceae e Rubiaceae (Giulietti et al. 2005). As demais espécies se referem às pteridófitas e gimnospermas (Shepherd 2000).

A enorme riqueza de espécies neotropicais está distribuída entre os diferentes hábitos vegetais existentes. Estima-se a existência de 22.000 espécies arbóreas (Center for Tropical Forest Science 2008), 29.000 epífitos (Gentry \& Dodson 1987a) e 9.000 lianas (Gentry 1991). Quando consideramos floras locais, contudo, a contribuição proporcional dos hábitos na riqueza de espécies varia consideravelmente. Árvores, arbustos e epífitas são mais ricos em áreas mais úmidas, enquanto o inverso parece acontecer para lianas e ervas (Gentry \& Dodson 1987b, Gentry 1991, Udulutsch 2004, Breier 2005, Rodrigues 2005). Além da umidade, a altitude também influencia a riqueza de diferentes hábitos (e.g. Gentry \& Dodson 1987a, Küper et al. 2004) ou de diferentes grupos, como as pteridófitas (cf. Dittrich et al. 2005).

Boa parte das espécies está concentrada na Floresta Ombrófila Densa (ou Floresta Pluvial Tropical), que no Brasil corresponde basicamente à Floresta Amazônica e à Floresta Atlântica (sensu Oliveira-Filho \& Fontes 2000). A Floresta Atlântica, em especial, é considerada como um dos mais importantes hotspots da biodiversidade mundial devido à sua elevada riqueza (cerca de 20.000 espécies) e ao seu alto nível de endemismo e de ameaça (Myers et al. 2000). Infelizmente, a Floresta Atlântica foi reduzida a menos de $16 \%$ de sua extensão original e a maior parte do que restou corresponde a fragmentos pequenos, isolados e alterados, geralmente sem qualquer proteção efetiva (Silva \& Tabarelli 2000, Ribeiro et al. 2009).

Tabarelli \& Mantovani (1999), trabalhando com espécies arbóreas no Estado de São Paulo, sugeriram que a importância da Floresta Atlântica na conservação de espécies vegetais estivesse mais relacionada aos níveis de endemismo do que à sua riqueza de espécies em escala local (i.e. diversidade alfa). Contudo, estudos realizados nos Estados do Espírito Santo e Bahia (um dos centros de endemismo da Floresta Atlântica - Mori et al. 1981) têm comprovado que a riqueza de espécies na Floresta Atlântica é comparável às áreas mais diversas do planeta, como Colômbia, Equador e Malásia (Martini et al. 2007). Os resultados encontrados por Thomas et al. (1998) e por Martini et al. (2007) confirmam esta alta riqueza e sugerem que áreas mais ao Sul da Floresta Atlântica teriam mesmo menor densidade de espécies arbóreas (mas ver Murray-Smith et al. 2008). Apesar do alto grau de endemismo da Floresta Atlântica, nenhuma família possui distribuição centrada na região fitogeográfica do Brasil Costeiro (Gentry 1982), exceto Myrtaceae (Mori et al. 1983). Contudo, são escassos os estudos que tratam sobre a distribuição das famílias e de suas espécies (e.g. Lima et al. 1997b, Amorim et al. 2008), que são informações básicas para compreender os padrões de distribuição geográfica atual e de suas mudanças ao longo do tempo (Prance 1987).
Este trabalho apresenta uma descrição dos padrões gerais de composição, diversidade de hábitos e distribuição geográfica das espécies vasculares da Floresta Ombrófila Densa do Parque Estadual Carlos Botelho (PECB), localizada na região sudeste do Brasil. Seu principal objetivo é comparar os padrões observados aos de outras florestas neotropicais, visando detectar possíveis particularidades da flora do PECB e auxiliar na definição de características diagnósticas da Floresta Atlântica do Sudeste do Brasil.

\section{Material e Métodos}

\section{1. Área de estudo}

O Parque Estadual Carlos Botelho (PECB), criado em 1982, possui 37.644 ha e está localizado entre as coordenadas geográficas $24^{\circ} 00^{\prime}$ e $24^{\circ} 15^{\prime} \mathrm{S}$, e $47^{\circ} 45^{\prime}$ e $48^{\circ} 10^{\prime} \mathrm{O}$, na região sul do Estado de São Paulo. O Parque cobre trechos de relevo montanhoso e escarpado da Serra de Paranapiacaba e os morros do Planalto de Guapiara (Ponçano et al. 1981), com altitudes variando entre 30 e $1.000 \mathrm{~m}$. A temperatura e precipitação anual média giram em torno de $22{ }^{\circ} \mathrm{C}$ e $1.600 \mathrm{~mm}$, respectivamente. Na parte baixa do Parque, o clima é subtropical úmido com verão quente ( $\mathrm{Cfa}$, segundo a classificação de Köppen), enquanto na parte alta o clima é subtropical úmido com verão fresco $(\mathrm{Cfb})$, com valores anuais médios de temperatura e precipitação relativamente mais baixos. Não há déficit hídrico significativo. A estação mais seca ocorre nos meses de Junho e Agosto. No PECB predominam rochas graníticas intrusivas, filitos e quartzitos (Almeida et al. 1981). Sobre estas, os Latossolos e Argissolos Vermelho-amarelos são os tipos de solo mais comuns, com elevados teores de matéria orgânica e de alumínio, baixos teores de bases trocáveis e elevada acidez (Pfeifer et al. 1986). O tipo vegetacional predominante do PECB, segundo o IBGE (Veloso 1992), é a Floresta Ombrófila Densa Montana e Submontana. Quanto à flora do $\mathrm{PECB}$, pode se afirmar que ela é bastante rica em número de espécies e relativamente bem conhecida quanto à sua composição (Lima et al. 2011). O PECB pertence à região fitogeográfica do Brasil Costeiro, a segunda mais rica em número de espécies no Neotrópico (Gentry 1982).

\section{Levantamento dos dados}

Este estudo utilizou como fonte de informações básica a lista de espécies disponível em Lima et al. (2011), que apresenta um checklist das espécies nativas florestais vasculares ocorrentes no PECB. Estes autores realizaram um extenso trabalho de compilação de informações e de revisão de nomenclatura que culminou em uma lista total com 1.143 espécies de 140 famílias botânicas, um total que representa uma boa amostra da flora do Parque e permite a identificação de padrões florísticos gerais.

Para cada uma das espécies citadas no checklist do PECB foram obtidas informações sobre o grupo taxonômico (pteridófitas, gimnospermas e angiospermas, subdivididas em monocotiledôneas e 'dicotiledôneas'), hábito (ou forma de vida) e distribuição geográfica. Por uma questão de praticidade, o termo dicotiledônea é usado aqui como uma referência ao grupo não-monofilético que inclui as espécies de angiospermas exceto as monocotiledôneas, ou seja, as "angiospermas basais", magnoliídeas e eudicotiledôneas (Angiosperm Phylogeny Group 2009). O hábito foi obtido através dos trabalhos de Klein (1979), Udulutsch (2004), Breier (2005), Ziparro et al. (2005), entre outros. Para as espécies não citadas nesta literatura, a obtenção do hábito foi feita através do conhecimento dos autores em seus respectivos grupos de especialidade ou através da observação e informações contidas em materiais de herbário. Adicionalmente, foram usados os dados de altura e diâmetro 
disponíveis em Aguiar (2003) e Rodrigues (2005) para a classificação das espécies de árvores e arbustos. Os hábitos considerados neste estudo foram: (i) herbáceo (ervas rasteiras, eretas ou subarbustos que não necessitam de outras plantas como suporte, incluindo epífitos acidentais e espécies rupícolas); (ii) arbustivo (até 3 metros de altura e caule ramificado); (iii) arbóreo (maiores que 3 metros, independentes de suporte); (iv) lianescente (espécies lenhosas ou herbáceas que dependem de suporte para seu desenvolvimento, mantendo sempre o contato do caule com o solo), subdivididas em escandentes (que se apóiam sobre outras), preênseis (com estruturas preensoras, como gavinhas ou ganchos) e volúveis (caules ou ramos se enrolam no forófito); (v) epifítico (plantas que se desenvolvem sem o contato caule-solo e que necessitam de outras plantas como suporte), subdividido em holoepífitos e hemiepífitos primários e secundários (inclui estranguladoras independentes de suporte quando adultas); e (vi) parasitas e hemiparasitas. Por vezes, mais de um hábito foi indicado para uma mesma espécie. Nestes casos, ambos foram citados na listagem final, mas o hábito considerado predominante para a espécie foi aquele usado durante as análises.

Quanto à distribuição geográfica, as espécies foram separadas de acordo com suas amplitudes de distribuição dentro das seguintes classes: pantropical (ocorrentes nos trópicos do novo e velho mundo); neotropical (América do Sul, América Central, Sul da América do Norte e Caribe); sul-americana (inclui distribuições disjuntas entre a Amazônia e a Floresta Atlântica); Floresta Atlântica (Floresta Atlântica lato sensu: Brasil, Paraguai e Argentina); Sul da Floresta Atlântica (distribuição no Sul e Sudeste Brasileiro, incluindo o Sul do estado da Bahia, Paraguai e Nordeste da Argentina); regional (distribuição restrita a, no máximo, três estados brasileiros); e local (espécies endêmicas da Serra de Paranapiacaba ou Vale do Ribeira). Estas classes foram definidas a partir dos padrões naturais de distribuição das espécies, mas em alguns casos o limite entre classes foi definido arbitrariamente (sul-americana e floresta atlântica). As informações de distribuição foram obtidas por meio de materiais depositados em herbários, várias revisões taxonômicas (em especial, os volumes da Flora Fanerogâmica do Estado de São Paulo, da Flora Neotropica e da Flora Ilustrada Catarinense) e das compilações de Barros et al. (1991), Mamede et al. (2001) e Sobral et al. (2006). Adicionalmente, foram consultados os checklists da flora das Guianas (Boggan et al. 1997), da Argentina (Argentina 2008) e da Estação
Biológica La Selva, na Costa Rica (La Selva Digital Flora 2008), além do no site do World Checklist of Selected Plant Families (2008). Espécies ocorrendo no PECB e, ao mesmo tempo, na Costa Rica ou nas Guianas tiveram suas distribuições enquadradas como neotropical ou sul-americana, respectivamente.

\section{Resultados}

\section{Composição e riqueza de espécies}

Para o total de espécies encontrado até o presente momento dentro do PECB (1.143 espécies), as dicotiledôneas apresentaram um número de espécies que foi quase três vezes maior que as monocotiledôneas e pteridófitas juntas (Tabela 1). Contudo, a participação destes grupos não foi constante entre os diferentes hábitos. Dicotiledôneas foram mais comuns entre árvores, arbustos e lianas, as monocotiledôneas e pteridófitas estiveram mais bem representadas entre os epífitos. As 10 famílias mais ricas em número de espécies foram Myrtaceae (85 espécies), Orchidaceae (81), Fabaceae (57), Asteraceae, Melastomataceae (54), Lauraceae (53), Rubiaceae (51), Bromeliaceae (43), Piperaceae (30) e Solanaceae (25). Os 10 gêneros mais ricos foram Eugenia (34 espécies), Ocotea (26), Leandra, Myrcia, Vriesea (18), Piper, Solanum (16), Miconia (14), Mollinedia (13) e Peperomia (12). Entre as pteridófitas (123 espécies), as cinco famílias mais ricas foram Polypodiaceae (23), Dryopteridaceae (18), Hymenophyllaceae (17), Aspleniaceae (11) e Cyatheaceae (nove), que juntas representam $63 \%$ das espécies de pteridófitas.

\section{Hábitos}

Uma comparação da contribuição dos hábitos no PECB com outras florestas Neotropicais é apresentada na Figura 1. Houve considerável variação na composição entre hábitos (Tabela 2), bem como no número de famílias representadas nos hábitos considerados. No PECB, apenas uma família teve representantes em todos os hábitos exceto parasitas: Rubiaceae. Outras cinco famílias tiveram representantes em quatro hábitos: Asteraceae, Fabaceae, Malvaceae, Melastomataceae e Urticaceae. Entre as pteridófitas, os epífitos foram os mais comuns ( 68 espécies ou $55 \%$ ), seguido pelas espécies terrícolas herbáceas (43 espécies) e terrícolas arborescentes (Alsophila spp. e Cyathea spp.). Apenas duas espécies de pteridófitas apresentaram hábito trepador (Lygodium volubile e Salpichaena volubilis). Entre

Tabela 1. Número de espécies vasculares em relação às diferentes hábitos ocorrentes no Parque Estadual Carlos Botelho, São Paulo, Brasil. Monocot $=$ monocotiledôneas; Dicot $=$ demais Angiospermas .

Table 1. Number of vascular species in respect to the different life forms found in the Carlos Botelho State Park, São Paulo, Brazil. Monocot=monocotyledons; Dicot $=$ others Angiosperms.

\begin{tabular}{|c|c|c|c|c|c|}
\hline \multirow{2}{*}{ Hábito } & \multirow{2}{*}{ Pteridófitas } & \multirow{2}{*}{ Gimnospermas } & \multicolumn{2}{|c|}{ Angiospermas } & \multirow{2}{*}{ Total } \\
\hline & & & Monocot & Dicot & \\
\hline Herbáceo & 44 & - & 58 & 108 & $210(18,4 \%)$ \\
\hline Arbustivo & 9 & - & 13 & 94 & $116(10,1 \%)$ \\
\hline Arbóreo & - & 1 & 3 & 443 & $447(39,1 \%)$ \\
\hline Lianas & 3 & - & 3 & 108 & $104(9,1 \%)$ \\
\hline Escandente & - & - & 1 & 18 & $19(1,7 \%)$ \\
\hline Preênsil & - & - & - & 38 & $38(3,3 \%)$ \\
\hline Volúvel & 3 & - & 2 & 42 & $47(4,1 \%)$ \\
\hline Epifítico & 67 & - & 137 & 52 & $256(22,4 \%)$ \\
\hline Holoepífito & 66 & - & 123 & 38 & $227(19,9 \%)$ \\
\hline Hemiepífito & 1 & - & 14 & 14 & $29(2,5 \%)$ \\
\hline Parasitas e Hemiparasitas & - & - & - & 10 & $10(0,9 \%)$ \\
\hline Total & 123 & 1 & 214 & 805 & 1143 \\
\hline
\end{tabular}


as angiospermas, o hábito arbóreo foi o mais rico e esteve presente no maior número de famílias (68), com algumas famílias sendo exclusivamente ou essencialmente arbóreas, como Annonaceae, Chrysobalanaceae, Lauraceae, Meliaceae, Myrtaceae e Sapotaceae.

O epifitismo vascular (incluindo hemiepífitos) foi o segundo hábito mais rico, com 256 espécies distribuídas em 29 famílias. Quanto à composição, Araceae, Bromeliaceae, Cactaceae, Gesneriaceae, Hymenophyllaceae, Orchidaceae e Polypodiaceae foram famílias predominantemente epifíticas. Os holoepífitos foram os mais comuns, seguido pelos hemiepífitos (e.g. Philodendron spp., Spirotheca rivierii, Coussapoa microcarpa, Oreopanax capitatus). O hábito herbáceo foi o terceiro mais rico em espécies no Parque

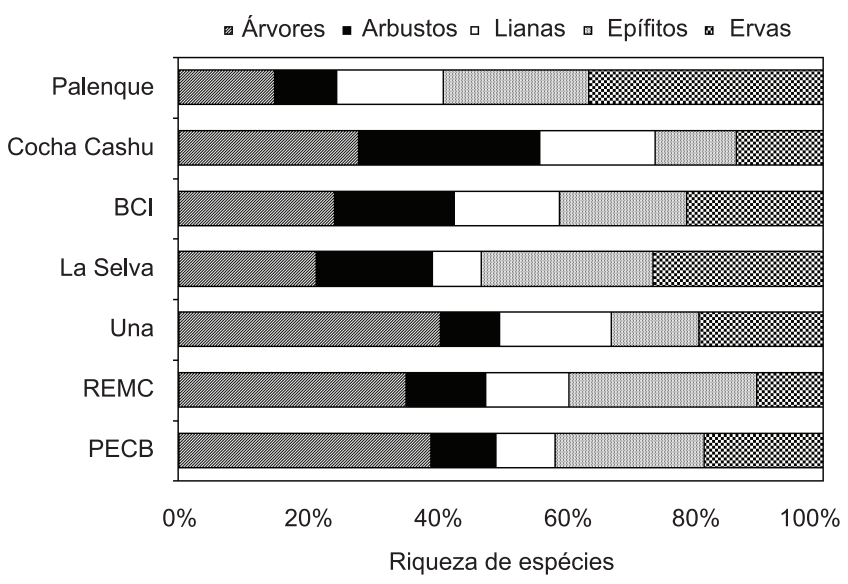

Figura 1. Riqueza proporcional dos hábitos em diferentes florestas neotropicais. Fonte: Palenque (Equador) $=$ Gentry \& Dodson (1987b); Cocha Cashu (Perú), Ilha de Barro Colorado (BCI, Panamá) e La Selva (Costa Rica $)=$ Gentry $(1990)$; Reserva Biológica de Una $($ Una, Brasil $)=$ Amorim et al. (2008); Reserva Ecológica de Macaé de Cima (REMC, Brasil)= Lima \& Guedes-Bruni (1997a); Parque Estadual Carlos Botelho (Brasil)= este estudo. Hemiparasitas incluídas entre os epífitos apenas para fins de comparação.

Figure 1. Proportional richness of life forms in different neotropical forests. Source: Palenque (Ecuador)= Gentry \& Dodson (1987b); Cocha Cashu (Peru), Barro Colorado Island (BCI, Panama) and La Selva (Costa Rica) $=$ Gentry (1990); Una Biological Reserve (Una, Brazil)= Amorim et al. (2008); Macaé de Cima Ecological Reserve (REMC, Brazil)= Lima \& Guedes-Bruni (1997a); Carlos Botelho State Park (Brazil) $=$ this study. Hemiparasites are included among the epiphytes for comparison only.
(210 espécies), ocorrendo em 62 famílias. Vinte e sete famílias foram exclusivamente herbáceas, com destaque para Cyperaceae, Commelinaceae, Gleicheniaceae e Marantaceae. Além destas, várias famílias tiveram grande proporção de espécies herbáceas como Asteraceae, Acanthaceae, Aspleniaceae, Begoniaceae, Blechnaceae, Dryopteridaceae, Melastomataceae, Poaceae e Rubiaceae. Os hábitos lianescente e arbustivo foram pouco representativos em número de espécies (104 e 116 espécies, respectivamente) e estiveram distribuídas em 30 e 23 famílias, respectivamente. Houve várias famílias exclusivamente lianescentes, dentre elas Aristolochiaceae, Convolvulaceae, Cucurbitaceae, Dilleniaceae, Dioscoreaceae, Menispermaceae, Passifloraceae e Vitaceae. Entre os arbustos, apenas uma família pode ser considerada exclusivamente arbustiva no PECB, Erythroxylaceae. O parasitismo foi o hábito menos diversificado no Parque, com apenas 10 espécies. As hemiparasitas (Psittacanthus spp., Struthanthus spp. e Phoradendron spp.) foram as mais comuns, tendo sido encontrada uma parasita de raiz, Lophophytum leandri.

\section{Distribuição geográfica}

Os padrões de distribuição geográfica das espécies do PECB (Tabela 3) evidenciaram que espécies comuns às floras da África e Ásia tropical são raras no PECB $(1,8 \%)$, sendo proporcionalmente mais comuns entre as pteridófitas. Ao contrário, a flora do PECB é caracterizada por um predomínio de espécies $(64,7 \%)$ endêmicas à Floresta Atlântica (valor inclui espécies com distribuição no Sul da Floresta Atlântica, regional e local). Dentre as espécies restritas à Floresta Atlântica, a distribuição predominante foi o Sul da Floresta Atlântica, caracterizada principalmente por espécies distribuídas no Sul e Sudeste do Brasil. Espécies distribuídas por toda a Floresta Atlântica (i.e. Sul ao Nordeste do Brasil) foram menos comuns que espécies de distribuição regional, fato que provavelmente deve-se a posição mais austral do PECB. Entre as espécies regionais, 22 estiveram restritas ao estado de São Paulo, enquanto 55 e 63 espécies ocorreram em dois (i.e. SP e RJ/PR/MG) e três estados brasileiros, respectivamente. A distribuição de apenas duas espécies foi citada como local: a bromélia Quesnelia violacea, restrita ao sul do estado e Handroanthus botelhensis (Bignoniaceae), árvore descrita através de materiais coletado dentro do Parque. De maneira geral, as famílias com maior número de espécies no PECB foram também aquelas com maior número de espécies endêmicas à Floresta Atlântica (Tabela 4).

Tabela 2. Famílias mais ricas, por hábito, encontradas para o Parque Estadual Carlos Botelho, São Paulo, Brasil. Ver texto para detalhes da separação das espécies em hábitos. Circunscrição em famílias segundo APG III (Angiosperm Phylogeny Group 2009).

Table 2. The most species-rich families in respect to the life form in the Carlos Botelho State Park, São Paulo, Brazil. See text for details on life form definition. Family circumscription followed APG III (Angiosperm Phylogeny Group 2009).

\begin{tabular}{|c|c|c|c|c|}
\hline Árvores & Arbustos & Epífitos & Ervas & Lianas \\
\hline Myrtaceae (85) & Melastomataceae (19) & Orchidaceae (74) & Asteraceae (20) & Apocynaceae (14) \\
\hline Lauraceae (53) & Asteraceae (14) & Bromeliaceae (43) & Cyperaceae (17) & Bignoniaceae (14) \\
\hline Fabaceae (37) & Piperaceae (14) & Polypodiaceae (22) & Melastomataceae (13) & Fabaceae (12) \\
\hline Rubiaceae (23) & Solanaceae (14) & Araceae (19) & Rubiaceae (12) & Asteraceae (8) \\
\hline Melastomataceae (21) & Rubiaceae (10) & Hymenophyllaceae (15) & Acanthaceae (8) & Malpighiaceae (8) \\
\hline Sapotaceae (14) & Cyatheaceae (9) & Cactaceae (14) & Begoniaceae (7) & Celastraceae (4) \\
\hline Asteraceae (13) & Arecaceae (6) & Piperaceae (13) & Dryopteridaceae (7) & Cucurbitaceae (4) \\
\hline Euphorbiaceae (13) & Erythroxylaceae (3) & Dryopteridaceae (11) & Lamiaceae (7) & Passifloraceae (4) \\
\hline Monimiaceae (11) & Urticaceae (3) & Gesneriaceae (9) & Orchidaceae (7) & Rubiaceae (4) \\
\hline Solanaceae (10) & Fabaceae (2) & Loranthaceae (7)* & Poaceae (7) & Sapindaceae (4) \\
\hline Annonaceae (9) & Monimiaceae (2) & Aspleniaceae (6) & Fabaceae (6) & Menispermaceae (3) \\
\hline
\end{tabular}

*Família composta exclusivamente por hemiparasitas, incluídas entre os epífitos apenas para fins de comparação. 
Plantas vasculares em uma Floresta Ombrófila Densa Atlântica

Tabela 3. Padrões de distribuição das espécies registradas no Parque Estadual de Carlos Botelho.

Table 3. Patterns of geographical distribution of the species recorded in the Carlos Botelho State Park.

\begin{tabular}{lccrrr}
\hline \multicolumn{1}{c}{$\begin{array}{c}\text { Padrão de } \\
\text { distribuição }\end{array}$} & Pteridófitas & Gimnospermas & \multicolumn{2}{c}{ Angiospermas } & \multicolumn{2}{c}{ Total } \\
\cline { 3 - 5 } & & & Monocot & 2 & 8 \\
Pantropical & 11 & - & 38 & 110 & $197(1,8 \%)$ \\
Neotropical & 49 & - & 36 & 118 & $174(15,2 \%)$ \\
Sul-americana & 19 & 1 & 19 & 85 & $108(9,4 \%)$ \\
Floresta Atlântica & 4 & - & 88 & 365 & $491(43,0 \%)$ \\
Sul da Floresta Atlântica & 38 & - & 28 & 109 & $139(12,2 \%)$ \\
Regional & 2 & - & 1 & 1 & $2(0,2 \%)$ \\
Local & - & - & 2 & 9 & $11(1,0 \%)$ \\
Sem informações* & - & - & & & \\
\hline
\end{tabular}

*Espécies com identificação apenas em gênero.

Tabela 4. Famílias com maior número de espécies endêmicas à Floresta Atlântica. Em parêntese, a proporção de espécies endêmicas em relação ao total de espécies registradas.

Table 4. Families with the larger number of endemic species from the Atlantic Forest. In parenthesis, the proportion of endemic species of the total number of species recorded.

\begin{tabular}{lccccc}
\hline \multicolumn{1}{c}{ Família } & $\begin{array}{c}\text { Floresta } \\
\text { Atlântica }\end{array}$ & $\begin{array}{c}\text { Sul da Floresta } \\
\text { Atlântica }\end{array}$ & Regional & Local & $\begin{array}{c}\text { Total de } \\
\text { endêmicas }\end{array}$ \\
\hline Myrtaceae & 6 & 51 & 15 & - & $72(84,7 \%)$ \\
Melastomataceae & 2 & 30 & 16 & - & $48(88,9 \%)$ \\
Orchidaceae & 14 & 23 & 10 & - & $47(58,0 \%)$ \\
Lauraceae & 3 & 31 & 10 & - & $44(83,0 \%)$ \\
Asteraceae & 2 & 32 & 5 & 1 & $39(72,2 \%)$ \\
Bromeliaceae & - & 25 & 4 & - & $34(79,1 \%)$ \\
Fabaceae & 5 & 22 & 4 & - & $31(54,4 \%)$ \\
Rubiaceae & 3 & 17 & 4 & - & $24(47,1 \%)$ \\
Solanaceae & 5 & 13 & 4 & - & $22(88,0 \%)$ \\
Piperaceae & 5 & 10 & 2 & - & $19(63,3 \%)$ \\
Apocynaceae & 5 & 11 & 3 & 1 & $18(85,7 \%)$ \\
Bignoniaceae & 5 & 8 & & $17(77,3 \%)$ \\
\hline
\end{tabular}

Contudo, a proporção de endemismo não foi a mesma entre elas. Myrtaceae, Melastomataceae, Lauraceae, Solanaceae, Apocynaceae e Bignoniaceae apresentaram proporções maiores de endemismo, enquanto Orchidaceae, Fabaceae e Rubiaceae apresentaram proporções menores.

\section{Discussão}

\section{Composição e riqueza de espécies}

A comparação do número de espécies e de famílias do PECB com outras florestas do Neotrópico mostra a importância do Parque na conservação da flora vascular neotropical. As famílias mais ricas encontradas no PECB foram basicamente as mesmas de outras regiões da Floresta Ombrófila Densa Atlântica do Sudeste Brasileiro (Barros et al. 1991, Lima \& Guedes-Bruni 1997a, Mamede et al. 2001, Ziparro et al. 2005, Kirizawa et al. 2007). A alta riqueza de Orchidaceae, Fabaceae, Melastomataceae, Rubiaceae e Piperaceae parece ser uma constante em florestas do Neotrópico. Entretanto, a alta riqueza proporcional de Myrtaceae parece ser uma particularidade das Florestas do Leste do Brasil (Mori et al. 1983, Peixoto et al. 2008, mas ver Hopkins 2005 para um alta riqueza de Myrtaceae na Amazônia). Na América Central, Amazônia e Nordeste do Brasil, as famílias Araceae, Chrysobalanaceae, Euphorbiaceae, Moraceae, e alguns gêneros (em especial Piper, Ficus, Pouteria, Psychotria e Inga) são mais bem representados (Gentry 1990). Na Floresta Atlântica do Sudeste, além de Myrtaceae, outras famílias e gêneros parecem ser mais diversificados, dentre elas Asteraceae e Bromeliaceae, e os gêneros Begonia, Leandra, Mollinedia e Vriesea, como encontrado no PECB (ver também Lima \& Guedes-Bruni 1997a). Talvez a maior riqueza destas famílias e gêneros seja um padrão comum a estas florestas, podendo ser usado como ferramenta na sua delimitação florística. Reforçando esta sugestão, o gênero Mollinedia parece ser menos rico na Floresta Atlântica do Nordeste (e.g. Amorim et al. 2008, Peixoto et al. 2008).

A flora pteridofítica do PECB (123 espécies) foi relativamente rica quando comparada às florestas da Ilha do Mel (107 espécies - Salino et al. 2005), Reserva Ducke (87 - Hopkins 2005) e a outras florestas neotropicais. Mas, é importante ressaltar que este grupo ainda não foi intensamente inventariado no PECB (Lima et al. 2011), sugerindo a existência de várias outras pteridófitas no Parque. Tal fato é reforçado pela elevada riqueza encontrada em áreas próximas, como constatado por Dittrich et al. (2005-81 espécies em 1 ha no Paraná), Salino \& Almeida (2008 - 212 táxons no Parque Estadual de Jacupiranga, São Paulo, com 150.000 ha) e A. Salino (dados não publicados - mais de 200 espécies em 800 ha de Floresta Ombrófila Densa no estado de São Paulo). Outras áreas no Sul e Sudeste do país, além de áreas no Nordeste (Amorim et al. 2008), 
apresentam riquezas consideravelmente mais baixas, sobretudo aquelas mais afastadas da faixa costeira, com exceção da Serra do Cuscuzeiro, em Analândia (113 espécies - Salino 1996). A composição e riqueza por família encontrada no PECB foi bastante próxima àquela encontrada em outras florestas do Sul e Sudeste do Brasil (Sylvestre 1997, Dittrich et al. 2005, Salino et al. 2005), com uma alta contribuição de pteridófitas epífitas, contribuição esta que foi superior a muitas outras florestas tropicais (Gentry \& Dodson 1987a). Aparentemente, os resultados sugerem menor riqueza de Thelypteridaceae em florestas mais elevadas (Sylvestre 1997, Dittrich et al. 2005), como encontrado aqui.

\section{Hábitos}

A contribuição das árvores na riqueza total do PECB foi comparativamente maior do que em outras florestas neotropicais (Gentry \& Dodson 1987b, Hammel 1990, Foster 1990, Foster \& Hubbell 1990), nas quais ervas, arbustos e lianas são mais diversificados. Este resultado é um provável efeito da maior concentração de trabalhos realizados no PECB envolvendo o hábito arbóreo (Lima et al. 2011). Futuros levantamentos direcionados aos hábitos menos inventariados no PECB são necessários para avaliar com segurança a riqueza e composição por hábito no interior do Parque. De qualquer maneira, os resultados obtidos confirmam a tendência de que espécies não-arbóreas representam de 50 a 70\% da riqueza de florestas tropicais (Gentry \& Dodson 1987b, Lima \& Guedes-Bruni 1997a, Galeano et al. 1998).

As famílias mais ricas de árvores foram basicamente aquelas listadas por Lima \& Guedes-Bruni (1997b), Tabarelli \& Mantovani (1999) e Oliveira-Filho \& Fontes (2000) para a Floresta Atlântica do Sudeste, com pequenas mudanças de ordenação. O mesmo é válido para a riqueza por gênero. Contudo, a riqueza de árvores encontrada (447 espécies) foi superior às demais áreas consultadas (Lima \& Guedes-Bruni 1997b, Gentry 1990, Gentry \& Dodson 1987b, Ziparro et al. 2005), exceto na Reserva Ducke (Hopkins 2005) e provavelmente na Reserva Biológica de Una (Amorim et al. 2008). A riqueza de árvores no PECB foi superior ao estimado para toda a Floresta Atlântica de encosta de São Paulo (432: Tabarelli \& Mantovani 1999), representando aproximadamente $24 \%$ da riqueza arbórea para toda a Floresta Atlântica do Sudeste (Oliveira-Filho \& Fontes 2000). Apesar de apenas uma família ter sido exclusivamente arbustiva no PECB (i.e. Erythroxylaceae), Melastomataceae, Piperaceae, Rubiaceae e Solanaceae estiveram mais bem representadas entre os arbustos, o que parece ser um padrão entre as florestas neotropicais (Foster \& Hubbell 1990, Hammel 1990, Andreata et al. 1997, Laska 1997, Hopkins 2005, Ziparro et al. 2005).

Do total de famílias de angiospermas com hábitos epifíticos citadas para os Neotrópicos (Gentry \& Dodson 1987a), o número de famílias encontrado no PECB representa aproximadamente $38 \%$. A riqueza de epífitos foi bastante elevada e comparável a outras florestas neotropicais (Gentry \& Dodson 1987a,b, Fontoura et al. 1997, Küper et al. 2004). Entretanto, este valor certamente não reflete a real riqueza de epífitos do PECB visto que Breier (2005) encontrou mais de 161 epífitos em uma área de apenas 10,24 ha do Parque. Por outro lado, o PECB encontra-se entre as faixas altitudinais que mais favorecem a manutenção de alta riqueza de epífitos (Küper et al. 2004). Nesse sentido, um levantamento direcionado a este hábito certamente aumentará a contribuição dos epífitos na riqueza total e revelará Orchidaceae como a família mais rica do PECB, como constatado em vários outros locais (Barros et al. 1991, Fontoura et al. 1997, Freiberg \& Freiberg 2000, Küper et al. 2004). Corroborando os resultados encontrados por Gentry \& Dodson (1987a), Fontoura et al. (1997), Freiberg \& Freiberg (2000) e Kersten \& Silva (2006), as famílias de angiospermas mais ricas entre as epífitas foram
Orchidaceae, Bromeliaceae e Araceae, que juntas perfizeram $51 \%$ de todos os epífitos do Parque. Outro resultado que parece ser um padrão em florestas neotropicais é a grande contribuição das pteridófitas e dos epífitos verdadeiros na riqueza de epífitos vasculares (Freiberg \& Freiberg 2000, Küper et al. 2004).

Entre as herbáceas, as famílias mais importantes do PECB também foram próximas àquelas de outras florestas (Hammel 1990, Foster \& Hubbell 1990, Andreata et al. 1997), apesar da menor riqueza de Poaceae. O número total de herbáceas foi relativamente baixo (210 espécies incluindo pteridófitas) se comparado a outras florestas Neotropicais (192-376 espécies - Gentry \& Dodson 1987b), mas relativamente maior se comparado a Reserva Ecológica de Macaé de Cima (161 espécies incluindo pteridófitas - Andreata et al. 1997, Sylvestre 1997). Como dito anteriormente, tal resultado deve ser interpretado com cautela devido ao grau de conhecimento deste hábito no PECB e ao fato de que o checklist do PECB inclui atualmente apenas espécies florestais. A inclusão de áreas não florestais do Parque (e.g. campos antrópicos e de altitude) certamente elevará a riqueza de herbáceas no PECB.

Entre as lianas, as 30 famílias encontradas representam cerca de $50 \%$ das famílias com espécies de lianas para todo o mundo (Prósperi et al. 2001). A proporção deste hábito no PECB (9\%) está aquém da média sugerida por Gentry (1991) para áreas tropicais (19\%), principalmente porque este autor não considerou espécies escandentes entre as lianas. Mesmo considerando que lianas lenhosas são menos ricas na Floresta Ombrófila Densa (Gentry 1991, Udulutsch 2004, Martins et al. 2008), tal resultado deve-se em parte ao pequeno conhecimento deste hábito no Parque (Lima et al. 2011). Além disso, as famílias mais ricas foram levemente distintas daquelas sugeridas por Kim (1996) para a Província Costeira da Floresta Atlântica e por Lima et al. (1997a). Famílias relativamente mal representadas entre as lianas do PECB foram Asteraceae, Sapindaceae e Convolvulaceae, sobretudo os gêneros Mikania, Serjania e Ipomoea, comuns em áreas alteradas e/ou não florestais. Contudo, outros estudos Neotropicais apontam resultados semelhantes aos apresentados aqui, com Bignoniaceae, Apocynaceae e Fabaceae entre as famílias de lianas mais ricas (Foster 1990, Foster \& Hubbell 1990, Morellato \& Leitão-Filho 1996, Gentry 1991, Prósperi et al. 2001). Como encontrado por Gentry (1991), famílias exclusivamente lianescentes contribuíram pouco no número total de lianas encontrado. Lianas volúveis foram mais abundantes (em especial, Apocynaceae, Malpighiaceae e Asteraceae), seguido das lianas preênseis (Bignoniaceae e Celastraceae) e escandentes (Fabaceae), uma tendência próxima ao observado por Lima et al. (1997a) em outro trecho de Floresta Ombrófila Densa Atlântica.

\section{Distribuição geográfica}

Os resultados mostraram que a maior parte da flora do PECB (65\%) é composta por espécies típicas da Floresta Atlântica lato sensu. Adicionalmente, a maior parte destas espécies está restrita as regiões Sul e Sudeste do Brasil, ao longo da Costa Atlântica. Este foi o mesmo padrão encontrado por Lima et al. (1997b) para outra Floresta Ombrófila Densa Atlântica no Rio de Janeiro. Contudo, a ocorrência de espécies com distribuição restrita ao Estado de São Paulo ou mais restritiva foi muito mais baixa (3\%) se comparado aos estudos de Thomas et al. (1998), Lima et al. (1997b) e Amorim et al. (2008), que encontraram valores sempre acima de $18 \%$. Isto reforça a sugestão de limites proposta por Prance (1987) para os centros de endemismo da Floresta Atlântica, que inclui o Rio de Janeiro, Espírito Santo e Bahia, mas não se estende até o Sul do Estado de São Paulo. Murray-Smith et al. (2008) sugerem centros de endemismo mais ao Sul (incluindo São Paulo, Paraná e Santa Catarina), mas apenas em áreas mais próximas ao mar, o que não é o caso do PECB. Além disso, 
o alto endemismo pontual encontrado por Lima et al. (1997b) deve-se, em parte, às florestas alto-montanas lá existentes, que são pouco extensas e que ainda não foram amostradas no PECB. O mesmo pode ser dito sobre os padrões encontrados por Murray-Smith et al. (2008), que incluem também áreas com florestas de terras baixas e restingas. Por outro lado, a proporção total de espécies endêmicas à Floresta Atlântica no PECB foi maior do que aquela encontrada por estes autores (45 e 47\% - Thomas et al. 1998, 57\% - Lima et al. 1997b, $40 \%$ - Amorim et al. 2008), confirmando o alto grau de endemismo da flora no Parque, mesmo que em uma escala mais abrangente.

A família Myrtaceae, além da grande riqueza de espécies, apresentou uma das maiores proporções de endemismo, fortalecendo a sugestão de que as florestas do Brasil Costeiro são de fato um importante centro de diversidade desta família (Mori et al. 1983, Amorim et al. 2008, Murray-Smith et al. 2008). Melastomataceae também merece destaque por ter sido aquela com maior número de espécies endêmicas locais, superando inclusive Myrtaceae. Destaque especial também para as famílias Monimiaceae, Gesneriaceae, Arecaceae, Begoniaceae e Symplocaceae que apresentaram no PECB apenas espécies endêmicas ao bioma. Dentre as famílias com proporções mais baixas de endemismo, várias são pteridófitas, como Blechnaceae, Pteridaceae e Gleicheniaceae que não apresentaram nenhuma espécie endêmica à Floresta Atlântica. Este resultado faz sentido pensando nos mecanismos de dispersão do grupo que são muito eficientes, garantindo a colonização dos continentes (Tryon 1972). Cyatheaceae foi a principal exceção entre as pteridófitas com quase $90 \%$ de endemismo (quatro espécies no Sul da Floresta Atlântica e uma regional).

As herbáceas foram mais comuns entre as espécies de distribuição pantropical e neotropical, muito provavelmente por seus mecanismos e estratégias de dispersão. Entre as herbáceas são comuns atributos como longas distâncias de dispersão, grande produção de frutos e dormência de sementes, que auxiliam a ampliação de suas distribuições. Dentre as demais distribuições (i.e. sul-americana, Floresta Atlântica, Sul da Floresta Atlântica e regional), foi observado um padrão no qual o hábito arbóreo foi o mais comum, seguido dos epífitos, arbustos, herbáceas e lianas. Deve-se lembrar, contudo, que as árvores representam o grupo melhor inventariado no PECB. Nesse sentido, os registros para os demais grupos podem ter um viés para as espécies de ampla distribuição, que geralmente são mais facilmente reconhecidas em campo e possuem maiores abundâncias. Inventários direcionados para famílias como Araceae, Gesneriaceae, Begoniaceae, Cactaceae, Bromeliaceae, Marantaceae e Polypodiaceae, famílias com altas proporções de epífitos e herbáceas endêmicas à Mata Atlântica, poderão indicar de maneira mais precisa as particularidades da flora do PECB.

\section{Agradecimentos}

Agradecemos ao programa de pós-graduação em Ecologia do Instituto de Biociências da USP pelo suporte financeiro e Adriana M.Z. Martini e dois revisores anônimos pelas contribuições em versões prévias deste manuscrito.

\section{Referências Bibliográficas}

AGUIAR, O.T. 2003. Comparação entre métodos de quadrantes e parcelas na caracterização da composição florística e fitossociológica de um trecho de floresta ombrófila densa no Parque Estadual "Carlos Botelho" - São Miguel Arcanjo, São Paulo. Dissertação de Mestrado, Universidade de São Paulo, Piracicaba.

ALMEIDA, F.F.M., HASUI, Y., PONÇANO, W.L., DANTAS, A.S.L., CARNEIRO, C.D.R., MELO, M.S. \& BISTRICH, C.A. 1981. Mapa Geológico do Estado de São Paulo. Instituto de Pesquisas Tecnológicas do Estado de São Paulo, São Paulo, v.1. (Série Monografias 6).
AMORIM, A.M., THOMAS, W.W., CARVALHO, A.M.V., JARDIM, J.G. 2008. Floristics of the Una Biological Reserve, Bahia, Brazil. Mem. New York Bot. Gard. 100:67-146.

ANDREATA, R.H., GOMES, M. \& BAUMGRATZ, J.F.A. 1997. Plantas herbáceo-arbustivas terrestres da Reserva Ecológica de Macaé de Cima. In Serra de Macaé de Cima: diversidade florística e conservação em Mata Atlântica (H.C. Lima \& R.R. Guedes-Bruni, eds.). Jardim Botânico do Rio de Janeiro, Rio de Janeiro, p.65-73.

ARGENTINA. Secretaría de Ambiente y Desarrollo Sustentable - SADS. 2008. www.ambiente.gov.ar/archivos/web/normativa/File/ANEXO1_ R578.pdf (último acesso em 03/2008).

ANGIOSPERM PHYLOGENY GROUP - APG. 2009. An update of the angiosperm phylogeny group classification for the orders and families of flowering plants: APG III. Bot. J. Linn. Soc. 161:105-121. http://dx.doi. org/10.1111/j.1095-8339.2009.00996.x

BARROS, F., MELO, M.M.R.F, CHIEA, S.A.C., KIRIZAWA, M., WANDERLEY, M.G.L. \& JUNG-MENDAÇOLLI, S.L. 1991. Caracterização geral da vegetação e listagem das espécies ocorrentes. In Flora Fanerogâmica da Ilha do Cardoso (M.M.R.F Melo, F. Barros, M.G.L. Wanderley, M. Kirizawa, S.L. Jung-Mendaçolli \& S.A.C. Chiea, eds.). Instituto de Botânica, São Paulo, v.1.

BOGGAN, J., FUNK, V., KELLOFF, C. HOFF, M. CREMERS, G. \& FEUILLET, C. 1997. Checklist of the plants of the Guianas (Guyana, Surinam, French Guiana). 2nd ed. Centre for the Study of Biological Diversity, Smithsonian Institution, Washington.

BREIER, T.B. 2005. O epifitismo vascular em florestas do Sudeste do Brasil. Tese de Doutorado, Universidade Estadual de Campinas, Campinas.

CENTER FOR TROPICAL FOREST SCIENCE - CTFS. 2008. Plots. Smithsonian Tropical Research Institute. http://www.ctfs.si.edu/doc/ plots/index.html (último acesso em 07/2008).

DITTRICH, V.A.O., WAECHTER, J.L. \& SALINO, A. 2005. Species richness of pteridophytes in a montane Atlantic rain forest plot of Southern Brazil. Acta Bot. Bras. 19(3):519-525. http://dx.doi.org/10.1590/S010233062005000300013

FONTOURA, T., SYLVESTRE, L.S., VAZ, A.M.S.F. \& VIEIRA, C.M. 1997. Epífitas vasculares, hemiepífitas e hemiparasitas da Reserva Ecológica de Macaé de Cima. In Serra de Macaé de Cima: diversidade florística e conservação em Mata Atlântica (H.C. Lima \& R.R. Guedes-Bruni, eds.). Jardim Botânico do Rio de Janeiro, Rio de Janeiro, p.89-101.

FOSTER, R. 1990. The floristic composition of the Barro Colorado Island forest. In Four Neotropical Forests (A.H. Gentry, ed.). Yale University Press, New Haven, p.85-98.

FOSTER, R. \& HUBBELL, S.P. 1990. The floristic composition of the Rio Manu floodplain forest, In Four Neotropical Forests (A.H. Gentry, ed.). Yale University Press, New Haven, p.99-111.

FREIBERG, M. \& FREIBERG, E. 2000. Epiphyte diversity and biomass in the canopy of lowland and montane forests in Ecuador. J. Trop. Ecol. 16:673-688. http://dx.doi.org/10.1017/S0266467400001644

GALEANO, G., SUÁREZ, S. \& BALSLEV, H. 1998. Vascular plant species count in a wet forest in the Chocó area on the Pacific coast of Colombia. Biodivers. Conserv. 7:1563-1575. http://dx.doi. org/10.1023/A:1008802624275

GENTRY, A.H. 1982. Neotropical floristic diversity: phytogeographical connections between Central and South America, Pleistocene climatic fluctuations, or an accident of the Andean orogeny? Ann. Mo. Bot. Gard. 69: 557-593. http://dx.doi.org/10.2307/2399084

GENTRY, A.H. 1990. Floristic similarities and differences between Southern Central America and Upper and Central Amazonia. In Four Neotropical Forests (A.H. Gentry, ed.). Yale University Press, New Haven, p.141-159.

GENTRY, A.H. 1991. The distribution and evolution of climbing plants. In The biology of vines (F.E. Putz \& H.A. Mooney, eds.). Cambridge University Press, Cambridge.

GENTRY, A.H. \& DODSON, C.H. 1987a. Diversity and biogeography of neotropical vascular epiphytes. Ann. Mo. Bot. Gard. 74(2):205-233. http://dx.doi.org/10.2307/2399395 
GENTRY, A.H. \& DODSON, C.H. 1987b. Contribution of nontrees to species richness of a tropical rain forest. Biotropica 19:149-156. http://dx.doi. org/10.2307/2388737

GIULIETTI, A.M., HARLEY, R.M., QUEIROZ, L. P., WANDERLEY, M.G.L. \& VAN DEN BERG, C. 2005. Biodiversity and Conservation of Plants in Brazil. Conserv. Biol. 19:632-639. http://dx.doi.org/10.1111/j.15231739.2005.00704.x

HAMMEL, B. 1990. The distribution of diversity among families, genera, and habit types in the La Selva flora. In Four Neotropical Forests (A.H. Gentry, ed.). Yale University Press, New Haven, p.75-84.

HOPKINS, M.J.G. 2005. Flora da Reserva Ducke, Amazonas, Brasil. Rodriguésia 56:9-25.

KERSTEN, R.A. \& SILVA, S.M. 2006. The floristic composition of vascular epiphytes of a seasonally inundated forest on the coastal plain of Ilha do Mel Island, Brazil. Rev. Biol. Trop. 54(3):935-942.

KIM, A.C. 1996. Lianas da Mata Atlântica do Estado de São Paulo. Dissertação de Mestrado, Universidade Estadual de Campinas, Campinas.

KIRIZAWA, M., SUGIYAMA, M., LOPES, E.A. \& CUSTODIO-FILHO, A. 2007. Flora da Reserva Biológica do Alto da Serra de Paranapiacaba, Santo André, São Paulo, Brasil. http://www.ibot.sp.gov.br/PESQUISA/ paranapiacaba/paranapiacaba.htm (último acesso em 08/2007).KLEIN, R.M. 1979. Ecologia da flora e vegetação do Vale do Itajaí. Sellowia 31:11-164.

KÜPER, W., KREFT, H., NIEDER, J., KÖSTER, N. \& BARTHLOTT, W. 2004. Large-scale diversity patterns of vascular epiphytes in Neotropical montane rain forests. J. Biogeogr. 31:1477-1487. http:// dx.doi.org/10.1111/j.1365-2699.2004.01093.x

LASKA, M.S. 1997. Structure of understory shrub assemblages in adjacent Secondary and Old Growth Tropical Wet Forests, Costa Rica. Biotropica 29:29-37. http://dx.doi.org/10.1111/j.1744-7429.1997.tb00003.x

LA SELVA DIGITAL FLORA. 2008. http://sura.ots.ac.cr/local/florula3/ index.htm (último acesso em 03/2008).

LIMA, H.C. \& GUEDES-BRUNI, R.R. 1997a. Diversidade de plantas vasculares na Reserva Ecológica de Macaé de Cima. In Serra de Macaé de Cima: diversidade florística e conservação em Mata Atlântica (H.C. Lima \& R.R. Guedes-Bruni, eds.). Jardim Botânico do Rio de Janeiro, Rio de Janeiro, p.29-39.

LIMA, H.C. \& GUEDES-BRUNI, R.R. 1997b. Plantas arbóreas da Reserva Ecológica de Macaé de Cima. In Serra de Macaé de Cima: diversidade florística e conservação em Mata Atlântica (H.C. Lima \& R.R. GuedesBruni, eds.). Jardim Botânico do Rio de Janeiro, Rio de Janeiro, p.53-64.

LIMA, H.C., LIMA, M.P.M., VAZ, A.M.S.F. \& PESSOA, S.V.A. 1997a. Trepadeiras da Reserva Ecológica de Macaé de Cima. In Serra de Macaé de Cima: diversidade florística e conservação em Mata Atlântica (H.C. Lima \& R.R. Guedes-Bruni, eds.). Jardim Botânico do Rio de Janeiro, Rio de Janeiro, p.75-87.

LIMA, M.P.M., GUEDES-BRUNI, R.R., SYLVESTRE, L.S., PESSOA, S.V.A. \& ANDREATA, R.H. 1997b. Padrões de distribuição geográfica das espécies vasculares da Reserva Ecológica de Macaé de Cima. In Serra de Macaé de Cima: diversidade florística e conservação em Mata Atlântica (H.C. Lima \& R.R. Guedes-Bruni, eds.). Jardim Botânico do Rio de Janeiro, Rio de Janeiro, p.103-123.

LIMA, R.A.F, DITTRICH, V.A.O., SOUZA, V.C., SALINO, A., BREIER, T.B. \& AGUIAR, O.T. 2011. Flora vascular do Parque Estadual Carlos Botelho, São Paulo, Brasil. Biota Neotrop. 11(4): http://www. biotaneotropica.org.br/v11n4/pt/abstract?inventory+bn01211042011

MAMEDE, M.C.H., CORDEIRO, I. \& ROSSI, L. 2001. Flora vascular da Serra da Juréia, município de Iguape, São Paulo, Brasil. Bol. Inst. Bot. 15:63-124.

MARTINI, A.M.Z., FIASCHI, P., AMORIM, A.M. \& PAIXÃO, J.L. 2007. A hot-point within a hot-spot: a high diversity site in Brazil's Atlantic Forest. Biodiver. Conserv. 16:3111-3128. http://dx.doi.org/10.1007/ s10531-007-9166-6
MARTINS, S.E., ROSSI, L., SAMPAIO, P.S.P. \& MAGENTA, M.A.G. 2008. Caracterização florística de comunidades vegetais de restinga em Bertioga, SP, Brasil. Acta Bot. Bras. 22(1): 249-274. http://dx.doi. org/10.1590/S0102-33062008000100024

MORELLATO, L.P.C. \& LEITÃO-FILHO, H.F. 1996. Reproductive phenology of climbers in a Southeastern Brazilian Forest. Biotropica 28(2):180-191. http://dx.doi.org/10.2307/2389073

MORI, S.A., BOOM, B.M., CARVALINO, A.M. \& SANTOS, T.S. 1983. Ecological Importance of Myrtaceae in an Eastern Brazilian Wet Forest. Biotropica 15:68-70. http://dx.doi.org/10.2307/2388002

MORI, S.A., BOOM, B.M. \& PRANCE, G.T. 1981. Distribution Patterns and Conservation of Eastern Brazilian Coastal Forest Tree Species. Brittonia 33:233-245. http://dx.doi.org/10.2307/2806330

MURRAY-SMITH, C., BRUMMITT, N.A., OLIVEIRA-FILHO, A.T., BACHMAN, S., MOAT, J., LUGHADHA, E.M.N. \& LUCAS, E.J. 2008. Plant diversity hotspots in the Atlantic Coastal Forests of Brazil. Conserv. Biol. 23:151-163. PMid:18950472. http://dx.doi.org/10.1111/j.15231739.2008.01075.x

MYERS, N., MITTERMEIER, R.A., MITTERMEIER, C.G., FONSECA, G.A.B. \& KENT, J. 2000. Biodiversity hotspots for conservation priorities. Nature 403:853-858. PMid:10706275. http://dx.doi. org/10.1038/35002501

OLIVEIRA-FILHO, A.T. \& FONTES, M.A.L. 2000. Patterns of floristic differentiation among Atlantic Forests in southeastern Brazil and the influence of climate. Biotropica 32:793-810.

PEIXOTO, A.L., SILVA, I.M., PEREIRA, O.J., SIMONELLI, M., JESUS, R.M. \& ROLIM, S.G. 2008. Tabuleiro Forests North of the Rio Doce: Their representation in the Vale do Rio Doce Natural Reserve, Espírito Santo, Brazil. Mem. New York Bot. Gard. 100:319-350.

PFEIFER, R.M., CARVALHO, W.A., SILVA, D.A., ROSSI, M. \& MENDICINO, L.F. 1986. Levantamento semidetalhado dos solos do Parque Estadual de Carlos Botelho, SP. Bol. Téc. Inst. Flor. 40:75-109.

PONÇANO, W.L., CARNEIRO, C.D.R., BISTRICHI, C.A., ALMEIDA, F.F.M. \& PRANDINI, F. 1981. Mapa Geomorfológico do Estado de São Paulo. Instituto de Pesquisas Tecnológicas do Estado de São Paulo, São Paulo, v.1. (Série Monografias 5).

PRANCE, G.T. 1987. Biogeography of neotropical plants. In Biogeography and quaternary history in tropical America (T.C. Whitmore \& G.T. Prance eds.). Claredon Press, Oxford, p.46-65.

PRÓSPERI, J., CABALLÉ, G. \& CARAGLIO, Y. 2001. Lianas and hemiepiphytes: distribution, development, and adaptations. Selbyana 22:197-212.

RIBEIRO, M.C., METZGER, J.P., MARTENSEN, A.C., PONZONI, F.J. \& HIROTA, M.M. 2009. The Brazilian Atlantic Forest: How much is left, and how is the remaining forest distributed? Implications for conservation. Biol. Conserv. 142:1141-1153. http://dx.doi.org/10.1016/j. biocon.2009.02.021

RODRIGUES, R.R. 2005. Parcelas permanentes em 40 ha de florestas do Estado de São Paulo: uma experiência multidisciplinar. Fapesp. (4 Relatório Temático do Projeto Parcelas Permanentes). Disponível em: $<$ http://www.lerf.esalq.usp.br/parrel2005.php>. Acesso em: nov 2007.

SOBRAL, M., JARENKOW, J.A., BRACK, P., IRGANG, B., LAROCCA, J. \& RODRIGUES, R.S. 2006. Flora arbórea e arborescente do Rio Grande do Sul, Brasil. RiMa, Novo Ambiente, São Carlos.

SALINO, A. 1996. Levantamento das pteridófitas da Serra do Cuscuzeiro, Analândia, SP, Brasil. Rev. Bras. Bot. 19(2):173-178. http://dx.doi. org/10.1590/S0102-33062008000400009

SALINO, A. \& ALMEIDA, T.E. 2008. Pteridófitas do Parque Estadual do Jacupiranga, SP, Brasil. Acta Bot. Bras. 22(4):983-991.

SALINO, A., SILVA, S.M., DITTRICH, V.A.O. \& BRITEZ, R.M. 2005. Flora pteridofítica. In História natural e conservação da Ilha do Mel (M.C.M. Marques \& R.M. Britez, orgs.). Editora UFPR, Curitiba, p.85-101.

SHEPHERD, G.J. 2000. Conhecimento e diversidade de plantas terrestres do Brasil. Secretaria de Biodiversidade e Florestas, Ministério do Meio Ambiente, Brasília. 
SILVA, J.M.C. \& TABARELLI, M. 2000. Tree species impoverishment and the future flora of the Atlantic Forest of northeast Brazil. Nature 404:7274. PMid:10716443. http://dx.doi.org/10.1038/35003563

SYLVESTRE, L.S. 1997. Pteridófitas da Reserva Ecológica de Macaé de Cima. In Serra de Macaé de Cima: diversidade florística e conservação em Mata Atlântica (H.C. Lima \& R.R. Guedes-Bruni, eds.). Jardim Botânico do Rio de Janeiro, Rio de Janeiro, p.41-52.

TABARELLI, M. \& MANTOVANI, W. 1999. Colonização de clareiras naturais na Floresta Atlântica no Sudeste do Brasil. Rev. Bras. Bot. 20(1):57-66. http://dx.doi.org/10.1590/S0100-84041997000100006

THOMAS, W.W., CARVALHO, A.M.V., AMORIM, A.M.A., GARRISON, J. \& ARBELÁEZ, A.L. 1998. Plant endemism in two forests in southern Bahia, Brazil. Biodiver. Conserv. 7:311-322. http://dx.doi. org/10.1023/A:1008825627656
TRYON, R.M. 1972. Endemic areas and geographic speciation in tropical American ferns. Biotropica 4(3):121-131. http://dx.doi. org/10.2307/2989774

UDULUTSCH, R.G. 2004. Composição florística da comunidade de lianas lenhosas em duas formações florestais do Estado de São Paulo. Dissertação de Mestrado, Universidade de São Paulo, Piracicaba.

VELOSO, H.P. 1992. Manual técnico da vegetação brasileira. IBGE, Rio de Janeiro. (Manuais técnicos de geociências, n.1).

WORLD CHECKLIST OF SELECTED PLANT FAMILIES - WCSP. 2008. The Board of Trustees of the Royal Botanic Gardens, Kew. Disponível em: <http://www.kew.org/wcsp/>. Acesso em: nov 2008.

ZIPARRO, V.B., GUILHERME, F.A.G., ALMEIDA-SCABBIA, R.J. \& MORELLATO, L.P.C. 2005. Levantamento florístico de floresta atlântica no sul do Estado de São Paulo, Parque Estadual Intervales, Base Saibadela. Biota Neotrop. 5(1): http://www.biotaneotropica.org.br/v5n1/ pt/abstract?inventory+BN02605012005

Recebido em 09/03/2010 Versão reformulada recebida em 27/02/2012 Publicado em 06/03/2012 